\title{
Vehicles Anti-collision System
}

\author{
Ajit Kumar \\ Shri Mata Vaishno \\ Devi University, Katra \\ Jammu and Kashmir \\ 182320
}

\author{
Ankit Jaiswal \\ Shri Mata Vaishno \\ Devi University, Katra \\ Jammu and Kashmir \\ 182320
}

\author{
Neha Jaiswal \\ Acropolis Institute of \\ Technology \& \\ Research,indore \\ ,Madhya Pradesh \\ 452001
}

\author{
Rahul Sharma \\ Shri Mata Vaishno \\ Devi University, Katra \\ Jammu and Kashmir \\ 182320
}

\begin{abstract}
To communicate between vehicles running on highway a wireless technology should be applied which will enable the potential to avoid collision. This paper discusses the method of sharing information of vehicles using laser beam detection. The speed and distance of the vehicles is obtained using BLINDER laser detector according to which commands of driving is decided. This system provides warning message on the basis of data obtained by detector. If distance is within the safety limit, alert tone is activated. While distance of vehicles crosses threshold distance, this system will reduce the speed automatically.
\end{abstract}

\section{General Terms}

Laser light detection, Road accidents

\section{Keywords}

Vehicles communication system, BLINDER laser detector, commands on driving.

\section{INTRODUCTION}

As a human being they always try to be compatible and comfortable with the nature. According to data provided by NHTSA(National Highway Traffic Safety Administration) there were 5,811,000 vehicle crashes reported in 2008 by police across US and 37,261 people killed and 2,346,000 people injured [1]. On the behalf of analyzing data of death, $02 \%$ deaths are occurring because of road accident. Each year nearly 1.3 million people die in road accidents which mean 3,287 deaths a day.

Everyone came across with the news of road accidents on highway frequently. Some of them are due to circumstantial error and some of them are to due to human error. So, circumstantial error can't resolved by it but human error can be resolved by this technique. Because of driver's inability to keenly observe the vehicles vicinity while driving accidents takes place. Road accidents have become very important issue in reduced. Many papers and research work is proposed regarding alarm system for safe driving.

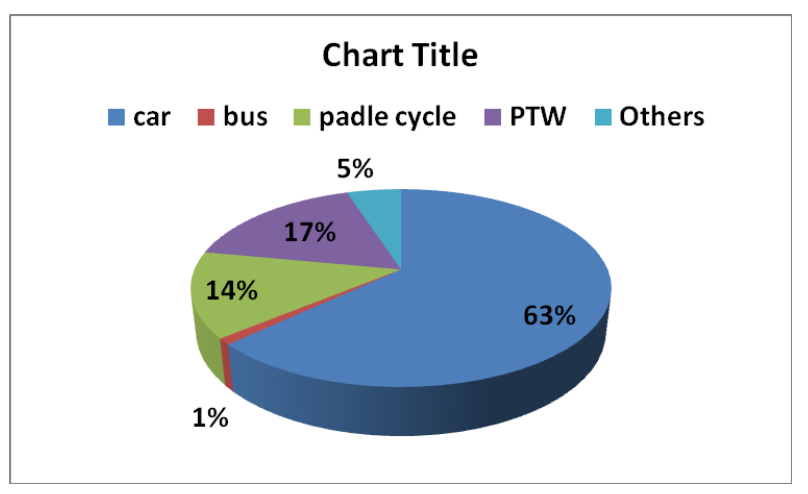

\begin{tabular}{|c|c|c|}
\hline Year & $\begin{array}{c}\text { Numbers of } \\
\text { Car crashes }\end{array}$ & $\begin{array}{c}\text { Per 100 } \\
\text { vehicles } \\
\text { traveled }\end{array}$ \\
\hline $\mathbf{2 0 1 2}$ & 25,580 & 1.14 \\
\hline $\mathbf{2 0 1 1}$ & 32,367 & 1.10 \\
\hline $\mathbf{2 0 1 0}$ & 32,885 & 1.11 \\
\hline $\mathbf{2 0 0 9}$ & 30,797 & 1.15 \\
\hline $\mathbf{2 0 0 6}$ & 38,648 & 1.42 \\
\hline $\mathbf{2 0 0 0}$ & 41,945 & 1.53 \\
\hline $\mathbf{1 9 9 5}$ & 41,817 & 1.73 \\
\hline $\mathbf{1 9 8 0}$ & 51,091 & 3.35 \\
\hline $\mathbf{1 9 7 0}$ & 52,627 & 4.74 \\
\hline
\end{tabular}

Figure 1: statistics record of road accidents

Vehicle detection system is used to decelerating the speed of vehicles as well as can be used to apply emergency brake. But emergency brake identification is another task so practically not feasible in this paper[2]. 


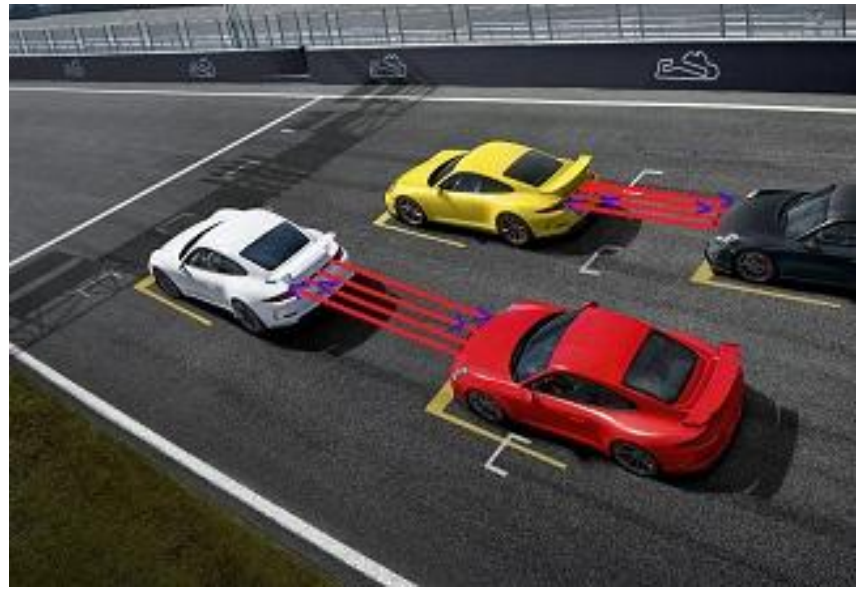

Figure 2: Identification of vehicles

As shown in figure 1, identification of vehicles using laser light (here two cars) is represented. A protocol therefore describes the syntax, semantics and synchronization of communication. A programming language describes the same for computations, so there is a close analogy between protocol and programming language: protocols are to communications while programming languages are to computations[3]. The AHS(Advance cruise-Assist Highway System) has been recently proposed that to avoid traffic accidents and traffic congestion states while communicating between vehicles via facilities installed on roads. But every communicating facilities could not be installed such as the vehicle information and communication system (VICS) because it requires some beacons along the roads[4].

\section{HISTORICAL BACKGROUND}

Inter-vehicular communications primarily as traffic and driver information systems incorporated in ATMS (Asynchronus Transfer Mode System) is treated as earliest research work conducted by JSK(Association of Electronic Technology for Automobile Traffic and Driving) of Japan in early 1980s (Tsugawa, 2005)[4].

The IST European Project CarTALK2000 was focusing on new driver assistance systems which are based upon intervehicle communication. The development of co-operative driver assistance systems and the development of a selforganizing ad-hocradio network as a communication basis with the aim of preparing a future standard[ 4.1].

American PATH (Hedrick et al., 1994) and European "Chaffeur" (Gehring et al.,1997) projects investigated and deployed automated platooning systems through the transmission of data among vehicles in 1990s to 2000. The FleetNet project in Germany (FleetNet project-Internet on the road, supported by six manufacturers and three universities from the 2000 though 2003, produced important results on several research areas, including the experimental characterization of VANETs, the proposal of novel network protocols (MAC, routing) and the exploration of different wireless technologies [3]

\section{SYSTEM OUTLINE}

This paper consists three major parts. First one is source of laser light beam which is used for producing laser beam. Second part of this system is BLINDER laser detector that will detect the reflection beam of laser light. The last and third part is alert and control System. In this part input of microcontroller is given by 'detector part which gives distance and speed of front coming obstacles. After comparing with input and stored value, output will be transferred to user.

\subsection{BLINDER (Laser detector)}

It detects infrared light of laser beam which is also a part of sunlight. The brighter the day, the more infrared light is scattered about which jammer/detector circuitry needs to content with. And in night due to absence of sun only infrared light presence makes easier. Therefore, this laser detector system will work best in full daylight or at night.

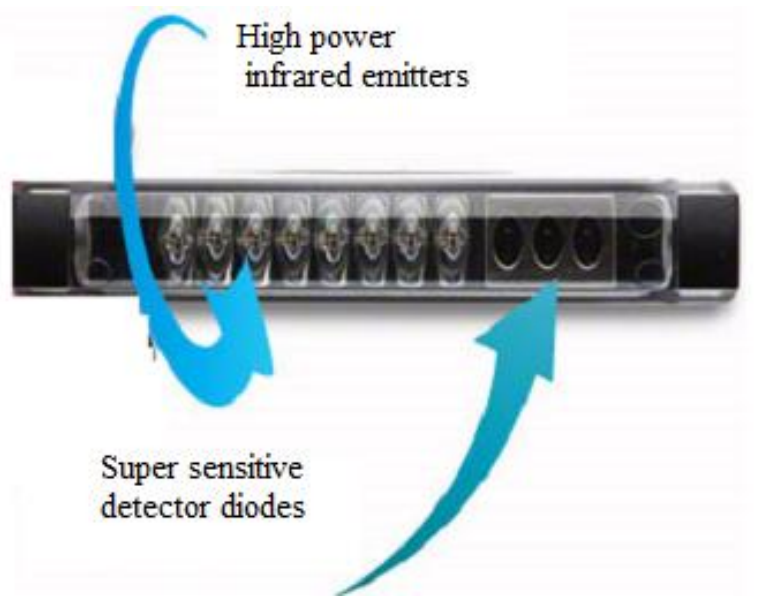

The BLINDER system will detect different objects with unequal effectiveness depending upon the color of the object. Bright objects reflect much better compared to dark, and so they will allow earlier detection and warning. The BLINDER system will not detect and alert on glass or other transparent materials. So, there should be cautious when parking close to shop windows and similar transparent objects [1]. The laser unit also will not respond to any incoming laser pulses in parking mode [1].

The BLINDER laser system contains transponder units and CPU module. The transponder units should be mounted such that transmitting and receiving infrared laser beam will not pass through any interfering objects. It is essential that view of the road for all transponders are clear and unobstructed, pointing straight ahead, mounted horizontal and parallel to the road surface and driving direction[1].

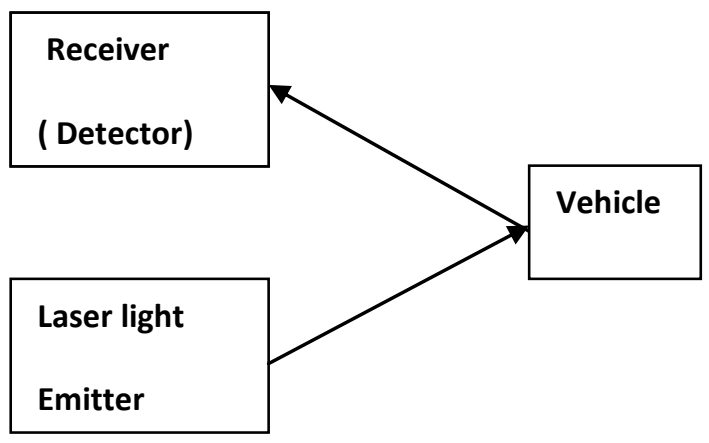

Figure 3: Performance principle of Blinder Laser detector

The following formula establishes the relationship between the distance up to the object $\mathrm{L}$ and reflecting time $\mathrm{T}$ is expressed by $\mathrm{L}=\mathrm{c} . \mathrm{T} / 2$, where $\mathrm{c}$ is the velocity of sound. That is, distance to the object can be ascertained by measuring the reflecting time involved in reaching the object. 


\subsection{WARNING SYSTEM}

Buzzer: Buzzer is used in a system to indicate or to grab the emergency attention occurred. Buzzer act as a panic horn which indicates the need of instant attention as in the condition goes haywire. As distance of detected vehicle is matched with critical distance (about $200 \mathrm{~m}$ ) buzzer should be turn on. In this way warning will be delivered.

\subsection{SPEED CONTROL}

DC motor control: DC motors are used to physically drive the application as per the requirement provided in software. The dc motor works on $12 \mathrm{v}$. to drive a dc motor, we need a dc driver called L293D. The dc motor driver is capable of driving two dc motors at a time. In order to protect the dc motor while from a back EMF generated by the dc motor while changing the direction of rotation, the $\mathrm{dc}$ motor driver have an internal protection suit. We can also provide the back EMF protection suit by connecting four diode configurations across each dc motor. DC motors power supply is dependent on distance between two vehicles. Motors are also turn off after particular distance.

\section{BLOCK DIAGRAM}

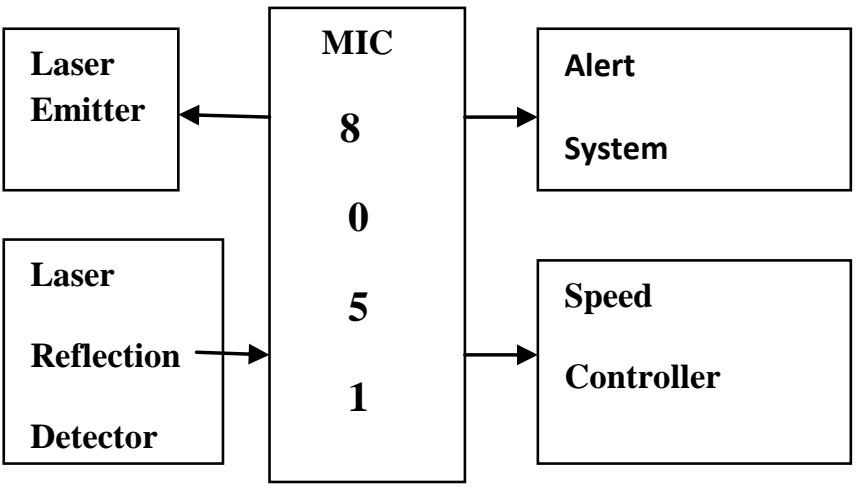

\section{WORKING}

As speed detection technology is going to be advance, there have also been advances in laser detection technology. The powerful sensors which can detect the light of laser guns from a far distance is BLINDER laser jammers/detectors. BLINDER can identify the incoming signal Within a few milliseconds.

Laser guns right out of "star wars" are now being used by Police the world over to enforce the speed limits.A laser speed gun measures the round-trip time for light to reach a car and reflect back. Using the number of pulses per second and identify through the use of lookup table the correct counter signal. Speed of light (from a laser speed gun) is very much faster than sound (speed of sound is $333 \mathrm{~m} / \mathrm{s}$ while speed of light is $984,000,000 \mathrm{~m} / \mathrm{s}$ in free space ). Laser speed gun emits a infrared laser light (of very short burst) and then waits for it to reflect off the vehicle. The gun counts the number of nanoseconds it takes for the round trip, and by dividing by 2 it can calculate the distance to the car.

For accurate aiming, lidar laser speed gun on a tripod takes 1,000 samples and calculate the speed of the car. For high accuracy gun takes several hundred samples over the course of a third of a second .The speed of a laser speed gun(for the police anyway) is that the size of the "cone" of light that the gun emits is very small, event at a range like 1,000 feet(300 meters).

\section{CONCLUSION}

Thus, this paper describes many functions related with obstacles distance detection and provides facility to control the vehicles collision due to some awareness. With the help of BLINDER it sense the reflection laser beam using which warning alarm and reduction in speed of vehicle is being done. In this way this system will be helpful in avoiding collision of vehicles. As reflection is detected it will first turn on alert system.

$\begin{array}{lrr}\text { Role } & \begin{array}{l}\text { People involved } \\ \text { in crashes }\end{array} & \text { Weighted \% } \\ \text { Drivers } & 3,943,244 & 66.5 \\ \text { Passengers } & 1,984,921 & 33.5 \\ \text { Unknown } & 1880 & 0.0 \\ \end{array}$

People involved in crashes by role of occupant [6]. After seconds of detection of obstacles, speed of vehicle is controlled by microcontroller 8051.Driver-related reasons of road accidents are classified into four parts recognition error $(41 \%)$, decision error (34\%), non performance error $(7 \%)$ like sleep, talking with friends while driving [6].

\section{REFERENCES}

[1] "BLINDER," n.d., para. 1 Retrieved from FAQs.p

[2] Kiyoshi Minami, Tohru Yasuma, Shigeru Okabayashi, Masao Sakata and Itsur Muramoto, Tadao Kohzu, "A collision- avoidance warning system using Laser Radar", SAE international paper,19.

[3] Biral F., Da Lio M., Lot R., Sartori R. (accepted). “An Intelligent Curve Warning System For Powered Two Wheel Vehicles". EUROPEAN TRANSPORT RESEARCH RE-VIEW, ISSN: 1866-8887A

[4] Nobuyoshi Mutoh, Yusuke Sasaki, "A Driver Assisting System for Eco-Vehicles with Motor Drive Systems Which Avoids Collision with Running Vehicles by Using Inter-Vehicle Communications", proceedings of the 2007 IEEE Intelligent Transportation Systems Conference Seattle, WA, USA, Sept. 30 - Oct. 3, 2007.

[5] Yusuke Takatori, Hiroyuki Yashima," A study of driving assistance system based on a fusion network of inter-vehicle communication and in-vehicle external sensors", 14th International IEEE Conference on Intelligent Transportation Systems Washington, DC, USA. October 5-7, 2011

[6] Bertolazzi E., Biral F., Da Lio M., Saroldi A., Tango F., "Supporting Drivers in Keeping Safe Speed and Safe Distance: the SASPENCE Subproject within the European Frame-work Program 6 Integrating Project PReVENT".

[7] Driver related reasons data, "National Highway Traffic Safety Administration(NHTSA)" July 2008.

[8] Jahnwi Tiwari, Ashish Kumar Singh,, Rakesh Kumar Jha and Kunal Kumar Singh. Article: Proposed Advanced Education System. International Journal of Computer Applications 91(17):1-4, April 2014 